Research Paper

\title{
MicroRNA-154-5p regulates the HPV16 E7-pRb pathway in Cervical Carcinogenesis by targeting CUL2
}

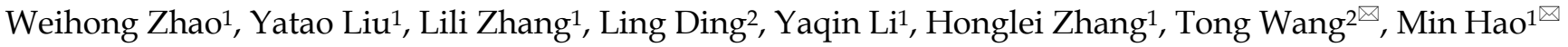 \\ 1. Department of Obstetrics and Gynecology, the Second Hospital of Shanxi Medical University, Taiyuan 030001, China. \\ 2. Department of Health Statistics, School of Public Health, Shanxi Medical University, Taiyuan, Shanxi, 030001, China. \\ $\square$ Corresponding authors: Tong Wang, Department of Health Statistics, School of Public Health, Shanxi Medical University, No. 86 Xinjiannanlu Street, Taiyuan \\ 030001, China; Tel: +86 3515 4066; E-mail: tongwang@sxmu.edu.cn. Min Hao, Department of Obstetrics and Gynecology, the Second Hospital of Shanxi Medical \\ University, No.182 Wuyi Road, Taiyuan 030001, China; Tel: +86 1393456 9668; E-mail: 2yuanhaomin@163.com.
}

(1) The author(s). This is an open access article distributed under the terms of the Creative Commons Attribution License (https://creativecommons.org/licenses/by/4.0/). See http://ivyspring.com/terms for full terms and conditions.

Received: 2020.03.11; Accepted: 2020.07.06; Published: 2020.07.11

\begin{abstract}
Cervical cancer, induced by persistent HPV infection, has a high mortality rate. The E3 ubiquitin ligase Cullin 2 (CUL2) is critical for HPV16 E7-mediated degradation of retinoblastoma protein (pRb). Dysregulation of microRNAs (miRNAs) is induced during tumorigenesis; however, the association between miRNA networks and CUL2, specific to cervical cancer, remains unknown. Herein, we determined miRNA profiles in cervical cancer tissues using an Affymetrix miRNA array. We found that miR-154-5p was downregulated during cancer progression using real-time quantitative reverse transcription PCR in 130 biopsy specimens. Bioinformatics analysis and dual-luciferase reporter assays indicated that miR-154-5p directly targets the CUL2 3'UTR. To determine the functional consequences of modulating miR-154-5p and CUL2 levels, HPV16-positive cervical cancer cell line ( $\mathrm{SiHa})$ was transfected with miR-154-5p mimic, miR-154-5p inhibitor, or CUL2 siRNA. The proliferation, migration, and invasion of transfected cells were evaluated using CCK8 cell counting kit, wound-healing assay, and Transwell invasion assay. Increased miR-154-5p expression promoted significantly reduced $\mathrm{SiHa}$ cell proliferation, migration, and invasion, whereas the miR-154-5p inhibitor had the opposite effect. CUL2 silencing had similar effects to those of the miR-154-5p mimic. Consistent with the inverse correlation between miR-154-5p and CUL2 levels, CUL2 silencing also increased pRb expression. To our knowledge, this is the first study to demonstrate that miR-154-5p regulates pRb expression by targeting CUL2 3'UTR, thereby playing a tumor-suppressive role in HPV16 E7-induced cervical carcinogenesis.
\end{abstract}

Key words: cervical cancer; CUL2; invasion; miR-154-5p; pRb; proliferation

\section{Introduction}

In China, cervical cancer has the highest incidence and mortality levels among female reproductive tract malignancies [1]. Squamous cell carcinoma (SCC), which is the predominant histological type, accounts for $80-85 \%$ of all cervical cancer cases. SCC develops from pre-existing non-invasive squamous precursor lesions referred to as cervical intraepithelial neoplasia (CIN). It has been confirmed that persistent infection with high-risk human papillomavirus (HR-HPV) is the primary cause of cervical cancer, and HPV16, in particular, is associated with more than $50 \%$ of SCC cases [2]. Although CIN can either progress or regress [3], only
$1 \%$ of the HPV-infected individuals might develop cervical cancer [4]. Therefore, the molecular mechanisms involved in HPV16 infection to the development of CIN and eventually to SCC remain largely unknown.

It is well established that HPV16 E7-dependent degradation of the retinoblastoma protein $(\mathrm{pRb})$ is a central carcinogenic pathway. Owing to a lack of intrinsic enzymatic activity, HPV16 E7 relies on protein-protein interactions to modulate the $\mathrm{pRb}$ degradation, especially interactions with the Cullin 2 (CUL2) ubiquitin ligase [5, 6]. Huh et al. found that HPV16 E7 binds to CUL2 and promotes $\mathrm{pRb}$ 
degradation, thereby causing malignant transformation of cells [5], while Todorovic et al. confirmed that the conserved region 3 of HPV16 E7 is a critical key for pRb degradation and CUL2 activity, supporting the finding that E7 and CUL2 interaction results in $\mathrm{pRb}$ degradation and leads to cell cycle alterations [7]. Therefore, CUL2-mediated regulation of HPV16 E7-induced pRb degradation is a critical step for the progression of HPV16 infection into cervical cancer.

miRNAs downregulate approximately a third of protein-coding genes in vivo through translational repression or gene silencing and are involved in various stages of viral infection and tumorigenesis [8]. However, reports on the correlation between miRNA expression and CUL2 regulation in the context of cervical cancer are rare. Our present study identified that miR-154-5p was significantly downregulated in SCC and its levels were negatively correlated with the proliferation and metastasis of $\mathrm{SiHa}$ cells. Mechanistic analyses revealed that miR-154-5p plays a tumor-suppressive role in cervical cancer through mediating pRb expression by targeting CUL2 3'UTR. Therefore, miR-154-5p may be a potential therapeutic target to block the progression of CIN into SCC caused by persistent HPV16 infections.

\section{Materials and Methods}

\section{Clinical specimens}

A total of 130 human cervical biopsy specimens including those from patients with only
HPV16-positive normal cervix $(\mathrm{n}=35)$, only HPV16-positive CIN1 ( $\mathrm{n}=31$ ), only HPV16-positive CIN2/3 ( $n=33)$, and only HPV16-positive SCC $(n=31)$ were collected at the Second Hospital of Shanxi Medical University between January 2016 and January 2018. According to the 2009 International Federation of Gynecology and Obstetrics (FIGO) staging standard, there were 12 cases of stage Ia, 7 cases of stage Ib, and 12 cases of stage IIa cancers. According to pathological classification, there were 9 cases of well-differentiated, 20 cases of moderately differentiated, and 2 cases of poorly differentiated cancers. 25 age-matched samples (SCC cases \pm 3 years) including 10 SCC tissues, 10 CIN3 tissues and 5 normal cervix tissues were selected out of total 130 patients for miRNA microarray analysis (Figure 1, see Table 1). All participants were Han Chinese who have lived in Shanxi for more than 5 years. Tumorigenesis was confirmed by two pathological examinations. Participants with leukemia, liver disease or other tumors were excluded. No patient received radiotherapy or chemotherapy before the tissues were obtained. After obtaining written informed consent from all participants, uterine cervix tissue biopsies were obtained by colposcopy, and the samples immediately stored in a $-80^{\circ} \mathrm{C}$ freezer. This study was approved by the Ethical Committees of the Second Hospital of Shanxi Medical University (IRB number: 2013-002).

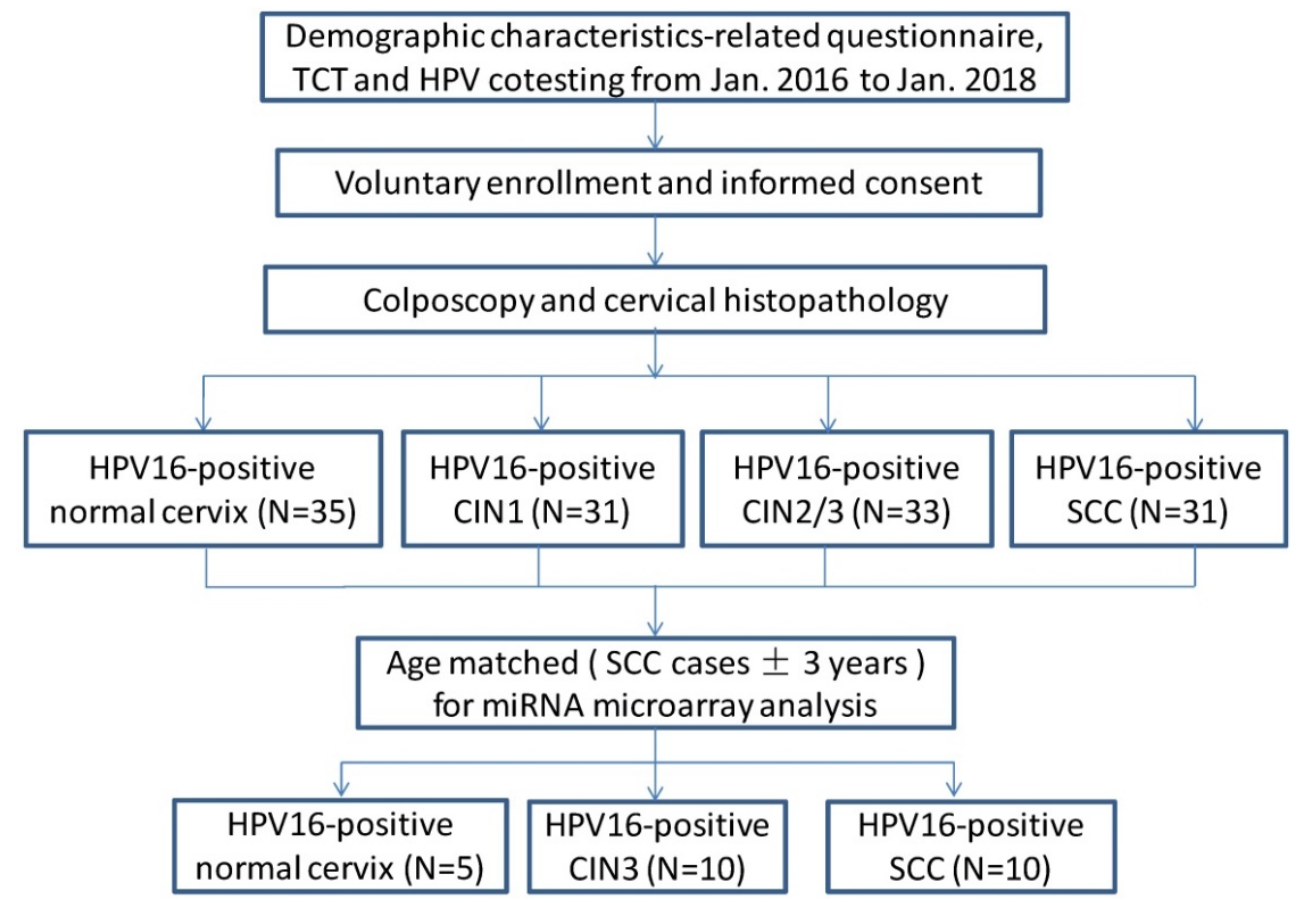

Figure 1. Study flow diagram. 
Table 1. Baseline characteristics of the subjects for microarray analysis

\begin{tabular}{llllll}
\hline Group & Sample NO. & Pathology & Age & HPV type & TCT \\
\hline 1 & 0000134 & Normal cervix & 46 & 16 & NILM \\
1 & 2100365 & Normal cervix & 44 & 16 & NILM \\
1 & 2100071 & Normal cervix & 47 & 16 & NILM \\
1 & 2100371 & Normal cervix & 43 & 16 & NILM \\
1 & 2100222 & Normal cervix & 45 & 16 & NILM \\
2 & 0000219 & CIN3 & 45 & 16 & ASCUS \\
2 & 2100218 & CIN3 & 50 & 16 & HSIL \\
2 & 2100209 & CIN3 & 46 & 16 & ASC-H \\
2 & 0000043 & CIN3 & 42 & 16 & ASCUS \\
2 & 2100399 & CIN3 with gland involvement & 50 & 16 & ASC-H \\
2 & 2100046 & CIN3 with gland involvement & 48 & 16 & NILM \\
2 & 0000126 & CIN3 & 43 & 16 & LSIL \\
2 & 2100005 & CIN3 & 46 & 16 & ASCUS \\
2 & 0000179 & CIN3 & 41 & 16 & ASCUS \\
2 & 2100068 & CIN3 & 43 & 16 & NILM \\
3 & 2100279 & SCC G2 & 48 & 16 & SCC \\
3 & 2100387 & SCC G2 & 49 & 16 & HSIL \\
3 & 2100418 & SCC G2 & 46 & 16 & ASCUS \\
3 & 0000266 & SCC G2 & 50 & 16 & ASCUS \\
3 & 0000172 & SCC G2 & 44 & 16 & SCC \\
3 & 2100125 & SCC G2 & 46 & 16 & ASCUS \\
3 & 2100385 & SCC G2 & 50 & 16 & HSIL \\
3 & 0000048 & SCC G2 & 47 & 16 & NILM \\
3 & 0000104 & SCC G2 & 50 & 16 & ASCUS \\
3 & 2100290 & SCC G2 & 49 & 16 & SCC \\
\hline
\end{tabular}

TCT, ThinPrep cytologic test; NILM, negative for intraepithelial lesion or malignancy; ASCUS, atypical squamous cells of undetermined significance ASC-H, atypical cells of undetermined significance, cannot exclude a high-grade squamous intraepithelial lesion; LSIL, low-grade squamous intraepithelial lesion; HSIL, high-grade squamous intraepithelial lesion; SCC, squamous cell carcinoma.

\section{MicroRNA microarray analysis and bioinformatics analysis}

Total RNA was extracted using Trizol ${ }^{\circledR}$ reagent (Life Technologies, Carlsbad, CA) and purified with an RNeasy ${ }^{\circledR}$ mini kit (Qiagen, Valencia, CA). Biotinylated cDNA was prepared from $250 \mathrm{ng}$ of total RNA using the Ambion ${ }^{\circledR}$ WT Expression Kit (Affymetrix, Santa Clara, CA) according to the standard Affymetrix protocol. Following biotinylation, fragmented cDNA was hybridized for $16 \mathrm{~h}$ at $45^{\circ} \mathrm{C}$ onto a GeneChip ${ }^{\circledR}$ miRNA 4.0 Array (Affymetrix, Santa Clara, CA). GeneChips were washed and stained in the Affymetrix Fluidics Station 450. All arrays were scanned using the Affymetrix ${ }^{\circledR}$ GeneChip Command Console, which was installed in the GeneChip ${ }^{\circledR}$ Scanner 3000 7G.

The prediction of miRNA target genes was performed using two public algorithms and associated databases (miRbase, available at http:// mirbase.org; and TargetScan version 7.1, available at http://targetscan.org). Gene ontology (GO) analysis was applied to analyze the main function of the target genes that had been classified according to the GO Project. In addition, pathway analysis was used to identify the key pathways of target genes according to the Kyoto Encyclopedia of Genes and Genomes (KEGG). Notably, candidate miRNAs that could potentially bind to the CUL2 3'UTR were identified, and binding affinity scores were calculated using TargetScan version 7.1.

\section{Dual-luciferase report assay}

The CUL2 3'UTR wild type fragment (CUL2WT) containing the predicted binding site of miR154-5 $\mathrm{p}$ and its corresponding mutant (CUL2-MUT), and the miR-154-5p inhibitor sponge (PC) were cloned by PCR into the pmirGLO vector (Promega, Madison, WI). The putative miR-154-5p binding site was mutated in the CUL2-MUT sequence by replacing 5'-AATATGACAGATAACCT-3' with 5'-TTTAACA CACTATTGGA-3'. 293T cells were co-transfected with pmirGLO-CUL2-WT, pmirGLO-CUL2-MUT, or pmirGLO-PC plus either miR-154-5p mimic negative control (NC). After incubating for $24 \mathrm{~h}$ and $48 \mathrm{~h}$, cells were collected for Firefly and Renilla luciferase activity measurements using the dual-luciferase reporter system (Promega, Madison, WI).

\section{Cell lines and transfection}

Two HPV16-positive human cervical cancer cell lines (SiHa and Caski) and 293T cells were purchased from the Cell Center of Shanghai Institutes for Biological Sciences. All cell lines were authenticated by the above-mentioned cell center by means of short-tandem repeat-polymerase chain reaction profiling. The cells were maintained in DMEM (Gibco, Carlsbad, CA) supplemented with 10\% FBS (Gibco). SiHa cells were transfected with miR-154-5p mimic, miR-154-5p mimic NC, miR-154-5p inhibitor, miR-154-5p inhibitor NC, CUL2 siRNA and CUL2 siRNA NC using Lipofectamine 3000 (Invitrogen, Carlsbad, CA) in accordance with the manufacturer's protocol. Blank control (where only the transfection reagent was added) and mock control were set up in all experiments. All nucleotides were FAM-labeled from GenePharma (Shanghai, China; see Table 2).

\section{Real-time quantitative reverse transcription PCR (RT-qPCR)}

Total RNA was isolated from cervical tissues or cell lines using the Eastep ${ }^{\circledR}$ Super Total RNA Extraction Kit (Promega, Madison, WI). cDNA was synthesized using Oligo (dT) and specific stem-loop primers with the PrimeScript ${ }^{\mathrm{TM}}$ RT reagent Kit (Takara, Kusatsu, Japan) added to a SYBR Premix containing Ex Taq ${ }^{\mathrm{TM}}$ (Takara, Kusatsu, Japan). The relative expression of miR-154-5p was calculated using the 2- $\Delta \Delta \mathrm{Ct}$ method with H-U6 as endogenous control. All specific primers were purchased from Sangon Biotech (Shanghai, China; see Table 2).

\section{Cell proliferation assay}

SiHa cells were seeded into a 96-well plate. At the indicated time post-seeding $(0,48,96 \mathrm{~h}), 10 \mu \mathrm{l}$ of 
Cell Counting Kit-8 (CCK-8; Dojindo Laboratories, Kumamoto, Japan) solution was added to each well. After further incubation for $1.5 \mathrm{~h}$, absorbance at 450 $\mathrm{nm}$ was recorded using a spectrophotometer (BioTek, Winooski, VT).

\section{Wound-healing assay}

SiHa cells were seeded into 6-well plates until they reached $90 \%$ confluency. A straight scratch was uniformly made in the center of each well with a micropipette tip. After scratching, cells were gently washed with PBS to remove the detached cells and maintained in serum-free DMEM. Cell migration was photographed and evaluated at $24 \mathrm{~h}$ and $48 \mathrm{~h}$ after scratching by light microscopy (Olympus, Tokyo, Japan).

\section{Transwell invasion assay}

Matrigel was diluted 1:5 with serum-free DMEM and added to the Transwell chambers (BD, Franklin Lakes, NJ) for incubation $3 \mathrm{~h}$. The cells were starved in serum-free DMEM for $12 \mathrm{~h}$ before $2 \times 10^{4}$ cells were seeded to each upper chamber inserted in a 24-well plate. $500 \mu 1$ of DMEM with $10 \%$ FBS was added to the lower chambers. After incubating for $24 \mathrm{~h}$, the noninvasive cells on the upper membrane were wiped with a cotton swab, and the invaded cells on the underside were fixed in methanol and stained with $0.1 \%$ crystal violet, then counted under a light microscope.

\section{Western blot analysis}

At $72 \mathrm{~h}$ following transfection, $\mathrm{SiHa}$ cells were collected and total protein extracted using RIPA lysis buffer (Beyotime Biotechnology, Shanghai, China). Conventional western blot analysis was performed using the primary antibodies anti-CUL2 (1:200, mouse; santa cruz; sc-166506), anti-pRb (1:200, mouse; santa cruz; sc-102) or anti- $\beta$-actin $(1: 1,000$, mouse; santa cruz; sc47778). After adding HRP-linked donkey anti-mouse IgG (1:2,000; abcam; ab97030), binding was detected using the Western Lightning Plus ECL reagent (Waltham, MA).

\section{Statistical analysis}

For microarray analyses, we used Student's $t$-test in the Limma R package (version 3.36.5) to filter for differentially expressed miRNAs. Empirical Bayesian moderation was used to correct the $P$ values. The threshold set was a fold change of $>2.0$ and $P<0.05$. The Fisher's exact test and Chi-square test were used to select the significant GO and pathway. The threshold set was $P<0.05$. Each in vitro experiment was performed in triplicate, and the results were presented as the mean value \pm standard error of mean. All statistical analyses were performed using the SPSS
16.0 software (IBM, Chicago, IL). Analysis of variance (ANOVA) or Kraskal-Wallis test with Post-hoc comparisons were used to establish the differences; $P<0.05$ was considered statistically significant.

Table 2. The sequences of primers and oligonucleotides used in this study

\begin{tabular}{ll}
\hline Primer/Oligonucleotide & Sequence (5'-3') \\
\hline miR-154-5p F & GCGCGCGTAGGTTATCCGTGTTG \\
miR-154-5p R & ATCCAGTGCAGGGTCCGAGG \\
miR-154-5p RT & GTCGTATCCAGTGCAGGGTCCGAGGTAT \\
& TCGCACTGGATACGACCGAAGG \\
H-U6 F & CTCGCTTCGGCAGCACA \\
H-U6 R & AACGCTTCACGAATTTGCGT \\
H-U6 RT & AACGCTTCACGAATTTGCGT \\
miR-154-5p mimic sense & UAGGUUAUCCGUGUUGCCUUCG \\
miR-154-5p mimic anti-sense & AAGGCAACACGGAUAACCUAUU \\
miR-154-5p mimic NC sense & UUCUCCGAACGUGUCACGUTT \\
miR-154-5p mimic NC anti-sense & ACGUGACACGUUCGGAGAATT \\
miR-154-5p inhibitor sense & CGAAGGCAACACGGAUAACCUA \\
miR-154-5p inhibitor NC sense & CAGUACUUUUGUGUAGUACAA \\
CUL2 siRNA sense & CCUCUUACUCAGGCUCCUUTT \\
CUL2 siRNA anti-sense & AAGGAGCCUGAGUAAGAGGTT \\
CUL2 siRNA NC sense & UUCUCCGAACGUGUCACGUTT \\
CUL2 siRNA NC anti-sense & ACGUGACACGUUCGGAGAATT
\end{tabular}

\section{Results}

miRNA expression profiles in different cervical tissues and bioinformatics analysis

A total of 77 miRNAs were differentially expressed in the three groups (Figure 2A, 2B, see Supplementary Table 1). The 77 differentially expressed miRNAs were searched in both miRbase and TargetScan to predict their target genes, and overlapping genes were used as the final dataset. A total of 2,618 target genes were predicted. GO analysis for highly enriched gene pathways identified the Wnt signaling pathway, the protein ubiquitination pathway (specifically, ubiquitin-dependent protein catabolic processes), the transforming growth factor beta receptor signaling pathway, as well as genes involved in regulation of epithelial to mesenchymal transition and regulation of transcription (Figure 2C). Moreover, KEGG analysis of the highly enriched gene pathways identified the phospholipase D signaling pathway, MAPK signaling pathway, mTOR signaling pathway, as well as genes involved in choline metabolism in cancer, and in glycerophospholipid metabolism (Figure 2D).

\section{CUL2 is a direct target of miR-154-5p}

Bioinformatics analysis predicted that the CUL2 3'UTR could potentially be targeted by the 17 differentially expressed miRNAs; among them, the miR-154-5p had the highest context percentile score (Figure 3A, see Table 3). miR-154-5p can potentially bind to two regions on CUL2 (Figure 3B), and it is highly conserved among different species (Figure 3C). 
These results suggest that miR-154-5p can form stable and close bonds with the CUL2 3'UTR.

Further, the dual-luciferase reporter assay showed that cells co-transfected with the miR-154-5p mimic and CUL2-WT plasmids had significantly less relative luciferase activity than cells co-transfected with the mimic NC and CUL2-WT plasmids $(P<0.05)$, whereas cells co-transfected with the miR-154-5p mimic and CUL2-MUT plasmids showed no significant reduction in the luciferase signal compared to the mimic NC $(P>0.05)$. Together, these results confirmed the direct binding between miR-154-5p and CUL2 (Figure 3D).

\section{Expression of miR-154-5p in different cervical tissues and cells}

The level of miR-154-5p expression in the CIN1, CIN2/3 and SCC were all lower than that in the normal cervix $(\mathrm{F}=129.83, P<0.001)$, consistent with the miRNA microarray analyses results (Figure 4A).

A

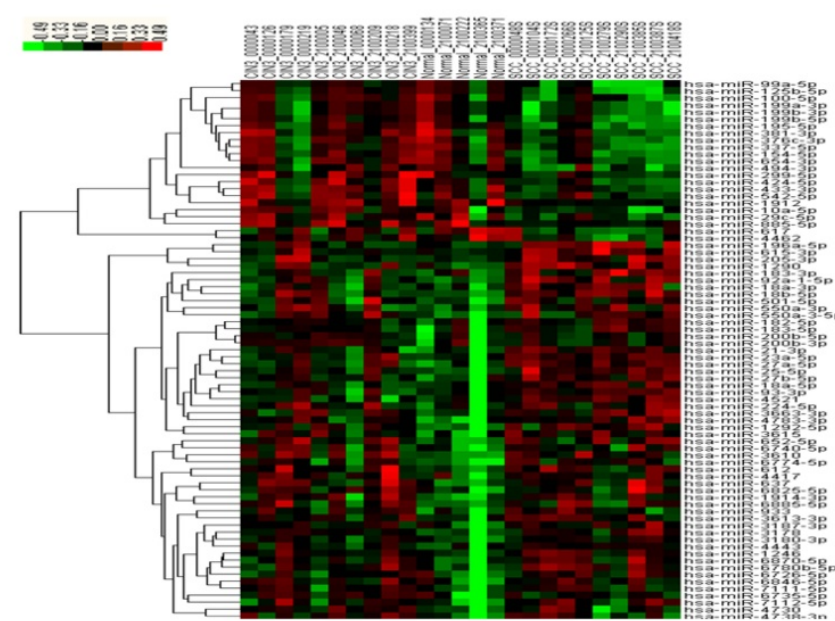

C

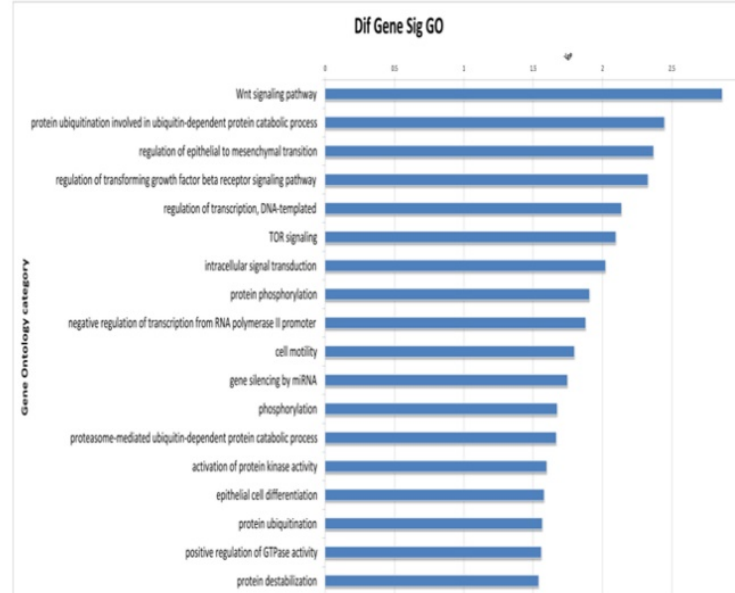

Further, miR-154-5p levels were also lower in the two human cervical cancer cell lines (SiHa and Caski) compared with 293T (Figure 4B).

\section{miR-154-5p suppressed proliferation, migration, and invasion of $\mathrm{SiHa}$ cells}

We used the miR-154-5p mimic or miR-154-5p inhibitor to transfect $\mathrm{SiHa}$ cells, and assessed the effect on proliferation, migration, and invasion. Transfection efficiencies were verified using fluorescence microscopy and by using RT-qPCR for the miR-154-5p levels. Fluorescence microscopic detection indicated transfection efficiencies over $90 \%$ in all groups (Figure 4C). The RT-qPCR results showed that miR-154-5p level was significantly increased following transfection with miR-154-5p mimic compared with mimic NC, while significantly decreased following transfection with miR-154-5p inhibitor compared with inhibitor NC (both $P<0.001$ ) (Figure 4D).

B

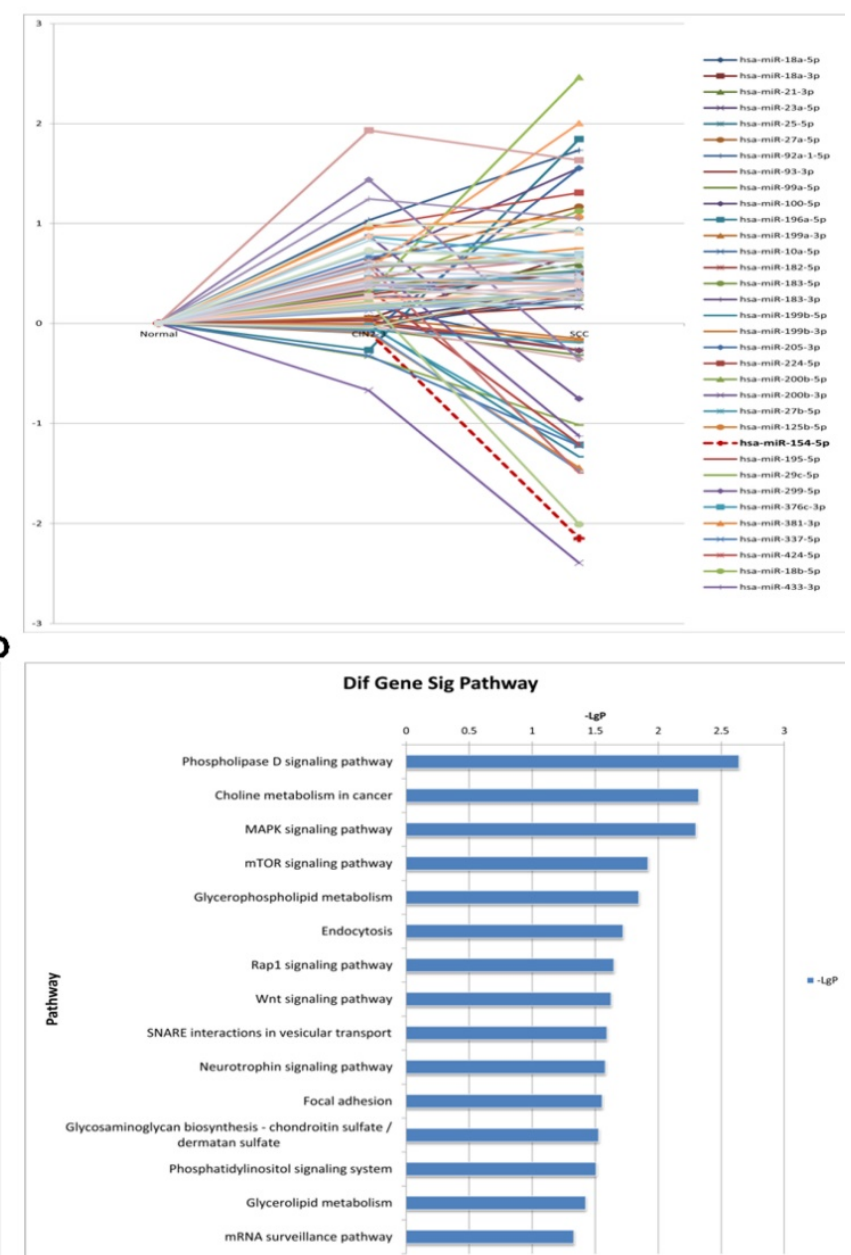

Figure 2. miRNA profiles in different HPV16 positive cervical tissues. (A) Hierarchical cluster of miRNA profiles. (B) The dynamic expression characteristics of miRNA profiles. (C) Log histogram of the significant GO. (D) Log histogram of the significant Pathway. 
Table 3. 3'-UTR of CUL2 was potentially targeted by 17 differentially expressed miRNAs in this study

\begin{tabular}{|c|c|c|c|c|c|c|}
\hline miRNA & Position in the UTR & seed match & context++ score & context++ score percentile & weighted context++ score & conserved branch length \\
\hline hsa-miR-93-3p & $705-711$ & $7 \mathrm{mer}-\mathrm{m} 8$ & -0.14 & 86 & -0.04 & 0.072 \\
\hline hsa-miR-199a-3p & $1605-1611$ & $7 \mathrm{mer}-1 \mathrm{~A}$ & -0.16 & 84 & -0.05 & 1.151 \\
\hline hsa-miR-183-3p & $551-557$ & 7 mer-m8 & -0.02 & 59 & -0.01 & 0.072 \\
\hline hsa-miR-199b-3p & $1605-1611$ & 7mer-1A & -0.16 & 84 & -0.05 & 1.151 \\
\hline hsa-miR-224-5p & $1620-1626$ & 7 mer-1A & -0.12 & 88 & -0.03 & 1.926 \\
\hline hsa-miR-27b-5p & $181-187$ & 7mer-1A & -0.2 & 89 & -0.2 & 1.671 \\
\hline \multirow[t]{2}{*}{ hsa-miR-154-5p } & $373-380$ & 8 mer & -0.44 & 99 & -0.44 & 5.685 \\
\hline & $1008-1015$ & 8mer & -0.21 & 93 & -0.06 & 0.851 \\
\hline hsa-miR-195-5p & $417-423$ & 7 mer-m8 & -0.34 & 97 & -0.27 & 8.638 \\
\hline hsa-miR-424-5p & $417-423$ & 7 mer-m8 & -0.41 & 98 & -0.33 & 8.638 \\
\hline hsa-miR-494-3p & $1268-1274$ & 7 mer-m8 & -0.02 & 51 & -0.01 & 0.192 \\
\hline hsa-miR-654-3p & $734-740$ & 7 mer-m8 & -0.03 & 49 & -0.01 & 0.075 \\
\hline hsa-miR-1290 & $1447-1454$ & 8 mer & -0.22 & 94 & -0.06 & 0.021 \\
\hline hsa-miR-1246 & $827-833$ & 7 mer-m8 & -0.14 & 86 & -0.04 & 0.021 \\
\hline hsa-miR-1914-3p & $1204-1210$ & 7 mer-m8 & -0.11 & 83 & -0.03 & 0 \\
\hline \multirow[t]{2}{*}{ hsa-miR-4443 } & $81-87$ & 7 mer-m8 & -0.28 & 94 & -0.28 & 0.901 \\
\hline & $949-955$ & 7mer-1A & -0.13 & 80 & -0.04 & 0 \\
\hline hsa-miR-4738-3p & $709-715$ & 7 mer-m8 & -0.02 & 44 & -0.01 & 0 \\
\hline hsa-miR-6825-5p & $920-926$ & 7mer-m8 & -0.23 & 78 & -0.06 & 0 \\
\hline
\end{tabular}

A

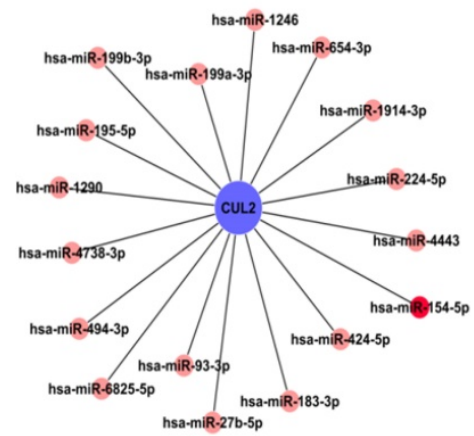

B

C

Position 373-380 of CUL2-3'UTR 5' UGUGCUUAGCAAUGC-AUAACCUA $3^{\prime}$ || ||||

hsa-miR-154-5p 3' GCUUCCGUUGUGCCUAUUGGAU 5'

Position 1008-1015 of CUL2-3'UTR 5' UGUGCUUAGCAAUGC - AUAACCUA 3' hsa-miR-154-5p 3' GCUUCCGUUGUGCCUAUUGGAU $\mathbf{5}^{\prime}$

miR-154 The same sequence and seed sequence

Human GAUAACCUAAUAAUUGUAUCUACAUU

Mouse GAUAACCUAAUAAUUGUAUCUACAUU

Rat GAUAACCUAAUAAUUGUAUCUACAUU

Rabbit GAUAACCUAAUAAUUGUAUCUACAUU

Chimp gaUAACCUAAUAAUUGUAUCUACAUU

Rhesus GAUAACCUAAUAAUUGUAUCUACAUU
D

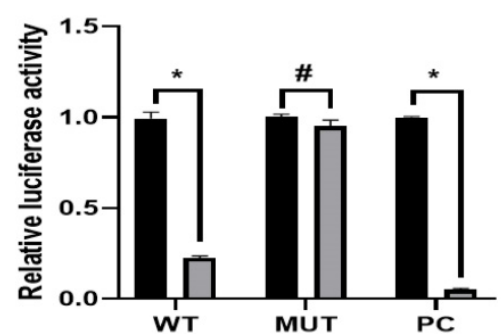

LUCI-293T-48h

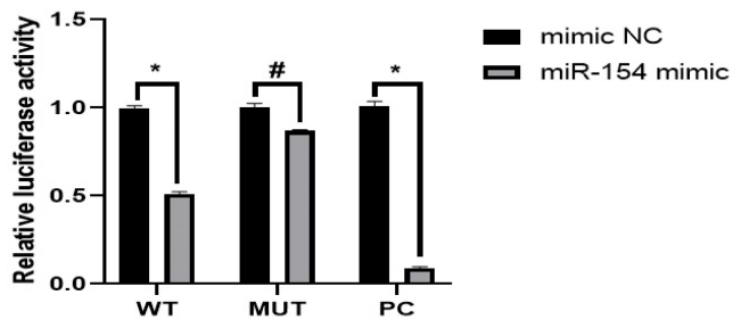

Figure 3. miR-154-5p can bind to CUL2 3'UTR directly. (A) Bioinformatics analysis predicted that CUL2 3'UTR was potentially targeted by 17 differentially expressed miRNAs. (B) The putative binding sites of CUL2 3'UTR and miR-154-5p. (C) miR-154-5p sequence is highly conserved. (D) Dual-luciferase report assays of cells cotransfected with miR-154-5p mimic and wild-type or mutant luciferase reporter. $(*, P<0.05$ versus corresponding NC).

Augmentation of miR-154-5p after transfection with its mimic reduced SiHa cells proliferation whereas miR-154-5p silencing increased cell proliferation, compared with the corresponding NC groups (both $P<0.05$, Figure 4E). Transfection with the miR-154-5p mimic inhibited the SiHa cells migration, whereas transfection with the miR-154-5p inhibitor enhanced cell migration compared to transfection with the corresponding NCs (both $P<0.05$, Figure $4 \mathrm{~F}$, $4 \mathrm{G})$. Compared with the corresponding NC, the invasive ability of $\mathrm{SiHa}$ cells transfected with miR-154-5p mimic decreased by $54.76 \%$, while the invasive ability of $\mathrm{SiHa}$ cells transfected with miR-154-5p inhibitor increased by $63.41 \%$ (both $P<0.05$, Figure $4 \mathrm{H}, 4 \mathrm{I})$. 
A

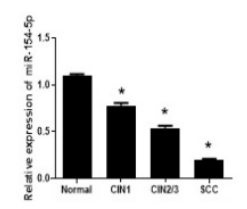

B

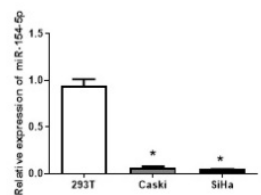

D

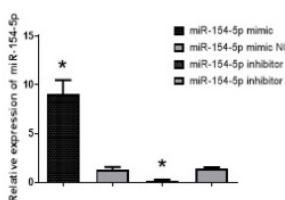

H
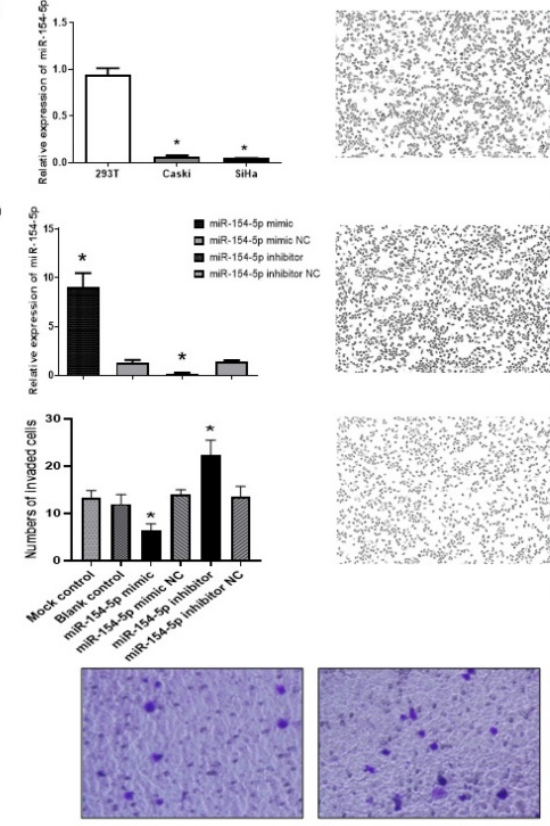

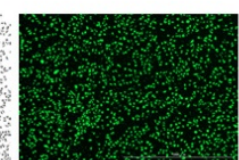

miR-154-5p mimic

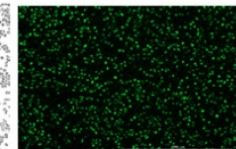

miR-154-5p mimic NC

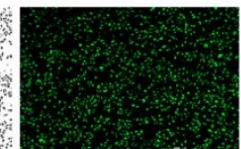

miR-154-5p inhibitor

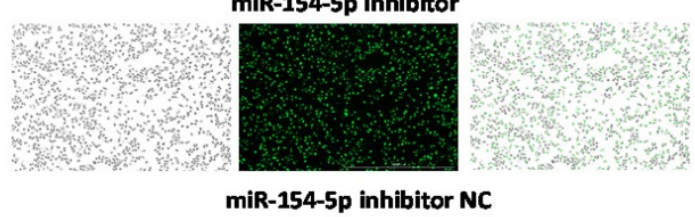

miR-154-5p inhibitor NC
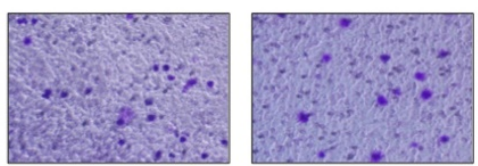

miR-154-5p mimic miR-154-5p mimic NC miR-154-5p inhibitor miR-154-5p inhibitor NC
$\mathbf{E}$

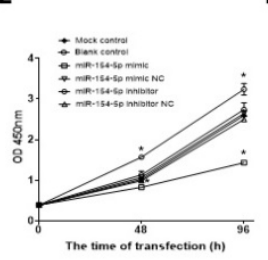

G
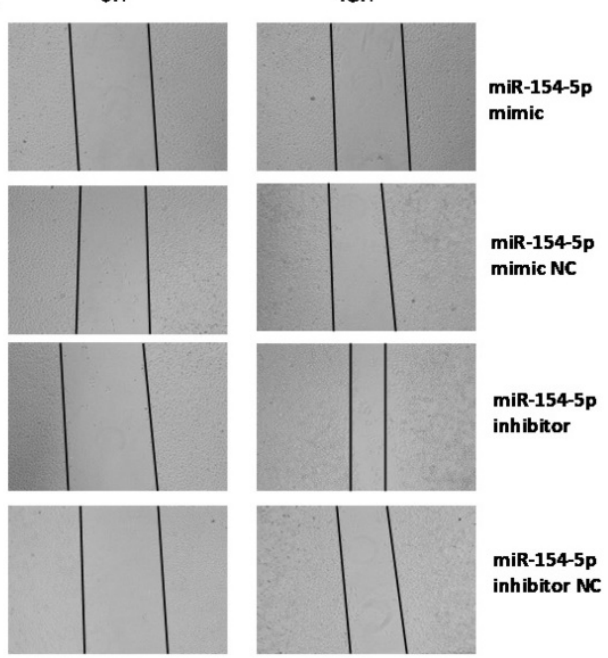

Figure 4. miR-154-5p was downregulated in cervical cancer tissues and effected SiHa cells proliferation, migration, and invasion. (A) miR-154-5p was downregulated in CIN and SCC tissues. (B) miR-154-5p was downregulated in cervical cancer cells. (C) SiHa cells were transfected with miR-154-5p mimic/inhibitor and corresponding NC. (D) Relative expression of miR-154-5p measured using RT-qPCR 48h post transfection. (E) miR-154-5p effected the SiHa cells proliferation. (F-G) miR-154-5p effected the SiHa cells migration. $(\mathrm{H}-\mathrm{I})$ miR-154-5p effected the SiHa cells invasion. (*, $\mathrm{P}<0.05$ versus corresponding NC).

\section{miR-154-5p mediated $p R b$ expression by targeting CUL2}

As described earlier, miR-154-5p can bind directly to the 3'UTR of CUL2. To further elucidate whether miR-154-5p would exert an inhibitory effect on CUL2 expression, western blot analysis was performed on transfected $\mathrm{SiHa}$ cells. At $72 \mathrm{~h}$ post-transfection, the expression of CUL2 protein in cells transfected with miR-154-5p mimic was lower than that of the mimic NC, while the expression of CUL2 protein in the miR-154-5p inhibitor-treated group was higher than inhibitor NC (both $P<0.05$, Figure 5A, 5B). In direct contrast to the changes observed with CUL2 expression, the expression of $\mathrm{pRb}$ in cells transfected with miR-154-5p mimic was higher than mimic $\mathrm{NC}$, while the expression of $\mathrm{pRb}$ in cells transfected with the miR-154-5p inhibitor was lower than inhibitor NC (both $P<0.05$, Figure 5C, 5D). In addition, Pearson correlation analysis showed an inverse correlation between the CUL2 and $\mathrm{pRb}$ expression $(r=-0.879, P<0.001$, Figure 5E).

\section{Knock-down of CUL2 suppressed proliferation, migration, and invasion of $\mathrm{SiHa}$ cells and increased $\mathbf{p R b}$ expression}

We transfected CUL2 siRNA into SiHa cells and assessed the effect on proliferation, migration, and invasion. Fluorescence microscopy detection showed that transfection efficiencies were over 90\% (Figure $6 \mathrm{~A})$. Western blot analysis showed that at $72 \mathrm{~h}$ following transfection, CUL2 protein levels were significantly decreased in cells transfected with CUL2 siRNA compared with siRNA NC $(P<0.001$, Figure 6B, 6C).

CUL2 protein knockdown reduced SiHa cells proliferation at both $48 \mathrm{~h}$ and $96 \mathrm{~h}$ following transfection with CUL2 siRNA (both $P<0.05$, Figure 6D). Further, CUL2 siRNA considerably inhibited SiHa cells migration when compared to the siRNA $\mathrm{NC}$ at $48 \mathrm{~h}$ post transfection $(P<0.05$, Figure $6 \mathrm{E}, 6 \mathrm{~F})$. The invasive ability of SiHa cells in the CUL2 siRNA group decreased by $46.34 \%$ compared with siRNA NC $(P=0.0112$, Figure 6G, 6H). At $72 \mathrm{~h}$ after transfection of SiHa cells with CUL2 siRNA, the $\mathrm{pRb}$ expression was higher than siRNA NC $(P<0.05$, Figure 6I, 6J). 


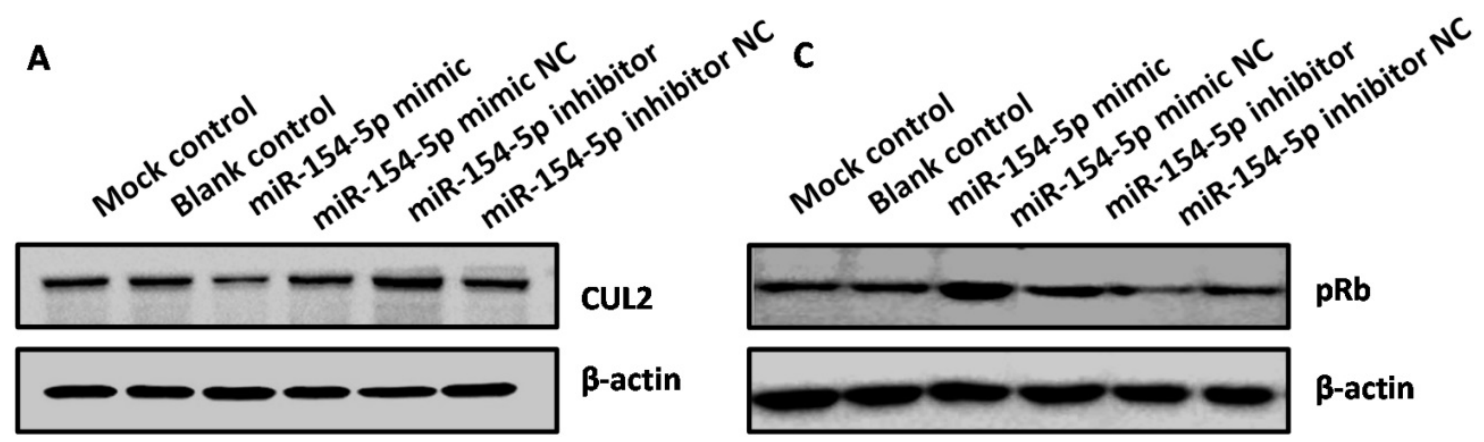

B

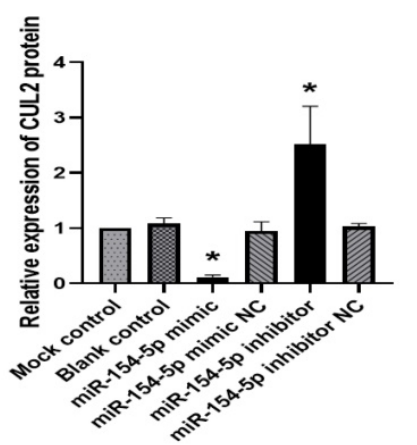

D

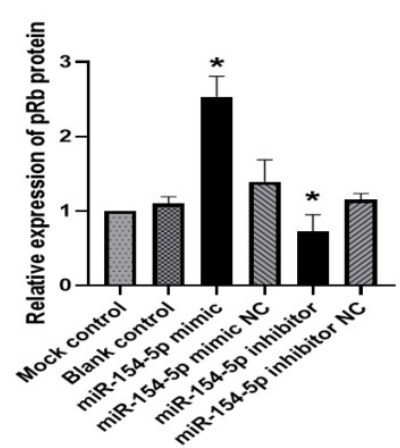

$\mathbf{E}$

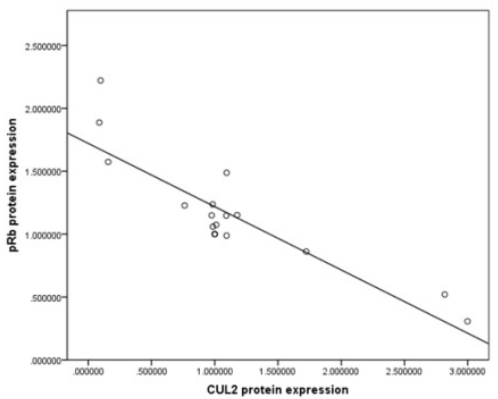

Figure 5. miR-154-5p effected the expression of CUL2 and pRb. (A-B) miR-154-5p regulated the expression of CUL2; (C-D) miR-154-5p regulated the expression of PRb; (E) Correlation analysis between CUL2 and pRb expression levels in SiHa. The relative expression of protein was measured by gray value assays. $\left({ }^{*}, \mathrm{P}<0.05\right.$ versus corresponding NC).

A
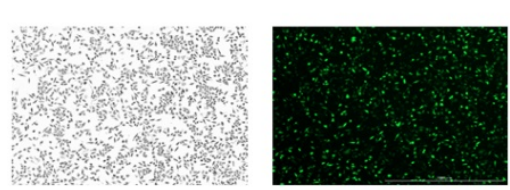

CUL2 SIRNA
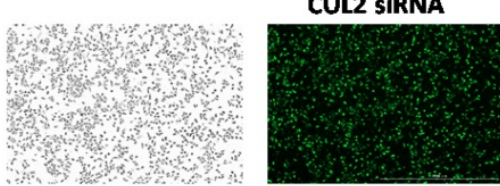

CUL2 SIRNA NC
B

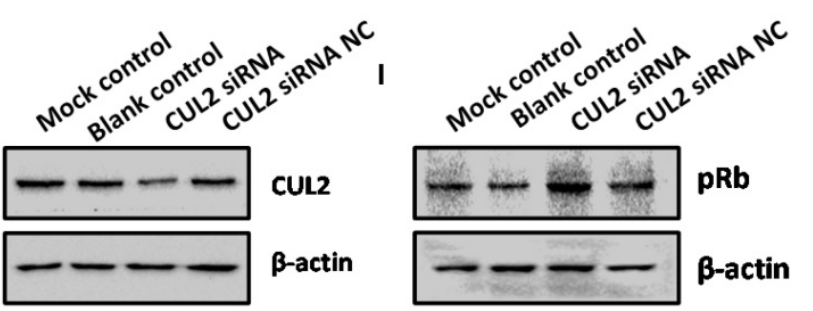

C

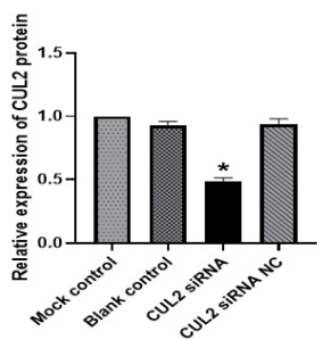

G

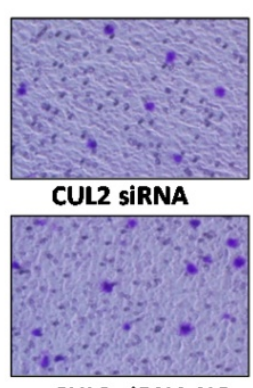

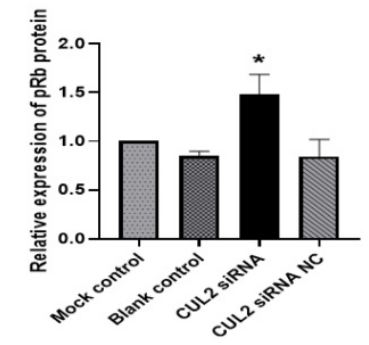

H

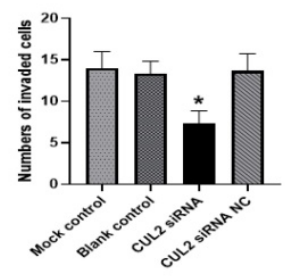

CUL2 SIRNA NC

Figure 6. CUL2 effected SiHa cells proliferation, migration, invasion, and the expression of pRb. (A) SiHa cells were transfected with CUL2 siRNA or siRNA NC; (B-C) The relative expression of CUL2 protein $72 \mathrm{~h}$ post transfection; (D) CUL2 effected the SiHa cells proliferation; (E-F) CUL2 effected the SiHa cells migration; (G-H) CUL2 effected the $\mathrm{SiHa}$ cells invasion; (I-J) CUL2 regulated the expression of $\mathrm{pRb}$ and the relative expression of protein was measured by gray value assays. $(*, \mathrm{P}<0.05$ versus corresponding NC). 


\section{Discussion}

Cervical cancer accounts for 570,000 new cases and 311,000 deaths worldwide annually, more than those of any other gynecological tumor [9]. SCC is the most prevalent cervical cancer and is closely associated with the carcinogenic HPV16 type. Nearly half the women with HPV16 infections persisting for at least two years developed precancer lesions within the subsequent five years [10]. The E6 and E7 genes are two known key oncogenes of HPV16. Compared to E6, more HPV16 E7 is required to sustain a malignant phenotype during an extensive characterization of primary cervical cancer HPV transcripts [11], making it a more potent driver for cervical cancer [12]. The E7 oncoprotein binds preferentially to the tumor suppressor $\mathrm{pRb}$ and disrupts the $\mathrm{pRb} / \mathrm{E} 2 \mathrm{~F}$ transcriptional repressor complex to initiate oncogenic transformation, leading to dysregulation of epithelial cell growth, which eventually leads to CIN progression, ultimately causing cervical cancer $[13,14]$. Therefore, blocking HPV16 E7-mediated $p R b$ degradation may inhibit the progression of CIN to SCC caused by persistent HPV16 infection.

The ubiquitin proteasome system is an important pathway for protein degradation in cellular organisms, which composed of ubiquitin, E1 ubiquitin-activating enzyme, E2 ubiquitin-binding enzyme, E3 ubiquitin ligase, and the proteasome. Cullin-Ring E3 ubiquitin ligases are the largest family of E3 ubiquitin ligases and are widely involved in the regulation of cyclins and transcription factor degradation [15]. Among these, CUL2 ubiquitin ligase complex has been shown to regulate cell cycle and tumorigenesis and become a hot topic of research [16, 17]. As the core component of the E3 ubiquitin-protein ligase complex, CUL2 has been predicted to be a tumor-suppressor since it causes the degradation of the a subunits of the pro-oncogenic hypoxia inducible factor [17]. In contrast, CUL2 has been reported to promote the progression of gastric cancer [18]. In the present study, we showed that inhibition of CUL2 expression significantly suppressed the oncogenic properties of SiHa cells. These results support an oncogenic role for CUL2 in the development of HPV16-induced cervical cancer, consistent with findings from a previous study [19]. In addition, we found a strong inverse correlation between the CUL2 and $\mathrm{pRb}$ levels in vitro. It has been reported that depletion of CUL2 by RNA interference increased the steady-state levels and stability of pRb in HPV16 E7-expressing cells rather than increasing translation of $\mathrm{pRb}$ [5]. Therefore, CUL2-mediated regulation of HPV16 E7-induced $\mathrm{pRb}$ degradation is a critical step for the progression of HPV16 infection into cervical cancer. However, the regulation of CUL2 is poorly understood.

The association between miRNA expression and cervical tumorigenesis has been investigated in epigenetic regulation studies. A systematic study of miRNA profiles from CIN to cervical cancer identified 42 up regulated and 21 down regulated miRNAs among different stages of cervical cancer development [20]. Approximately $50 \%$ of miRNA genes are located in fragile sites that are preferential targets for HPV16 integrations in cervical tumors [21]. Based on this, miRNAs play a synergic role in HPV16 infection leading to cervical cancer. Recent studies have found that in cervical cancer cells, miR-424 binds to CUL2 mRNA, and that overexpression of miR-424 decreases CUL2 mRNA and protein expression, thereby inhibiting cell proliferation, migration and invasion, promoting cell cycle arrest in the G1/S phase [19]. This suggests that the miRNA-mediated CUL2 regulation is an important target for blocking cervical cancer. However, reports on the correlation between miRNA expression and CUL2 regulation in the context of cervical cancer are rare. Therefore, insight into the regulatory networks of miRNA that target CUL2 during CIN progression may help to identify their contribution to the pathogenesis of cervical cancer.

In this study, we have identified a total of 77 miRNAs differentially expressed in CIN3 and SCC tissues compared with normal cervix tissues. According to GO analysis, "protein ubiquitination involved in ubiquitin-dependent protein catabolic processes" (GO: 0042787) had the second highest enrichment score, suggesting that this pathway is important for regulating protein levels and function during the development of HPV16-induced cervical cancer. The CUL2 scaffold protein is required for ubiquitin-dependent protein degradation [22]. In our study, bioinformatics analysis predicted that the CUL2 3'UTR was potentially targeted by the 17 differentially expressed miRNAs, among which miR-154-5p had the highest binding context score. Furthermore, the dual-luciferase reporter assay confirmed that miR-154-5p directly targeted CUL2 through its 3'UTR. Our findings indicate that CUL2 is a downstream target of miR-154-5p.

miR-154-5p is located on human chromosome $14 \mathrm{q} 32$ and is involved in the pathophysiology of various disorders [23]. Recently it has been shown that miR-154-5p, which is involved as a suppressor in the regulation of cancers, was downregulated in breast cancer, colorectal cancer, glioma, hepatocellular cancer, osteosarcoma, nasopharyngeal carcinoma, and non-small cell lung cancer [24-31]. Intriguingly, Lin et 
al. revealed that miR-154-5p was up regulated in renal cell carcinoma and acted as an oncogene with poor prognosis [32]. These results suggest that miR-154-5p may play a tumor-suppressive or oncogenic role, depending on the tumor types. Nevertheless, there are no studies on the expression of miR-154-5p and its role in cervical cancer. To our knowledge, this is the first report which shows that miR-154-5p expression was significantly reduced in CIN and SCC tissues. Therefore, we further investigated the miR-154-5p functions in the progression of cervical cancer. Our data showed that enforced expression of miR-154-5p significantly reduced the proliferation, migration, and invasion ability of SiHa cells. Conversely, inhibition of miR-154-5p had the opposite effect. Thus, the downregulation of miR-154-5p may be one of the factors that acts in coordination with HPV16 infection to induce cervical carcinogenesis or cervical cancer progression.

miRNA functions are observed through their effects on specific target genes. A recent study showed that miR-154-5p inhibits glioma cell proliferation, migration, and invasion by regulating PIWIL1 genes [29]. In colorectal cancer, miR-154-5p inhibits tumor cell proliferation and migration by blocking CCND2 [33]. However, the target gene(s) of miR-154-5p in cervical cancer have not yet been identified. As mentioned previously, we used bioinformatics analysis to predict and the dual-luciferase reporter assay to confirm that CUL2 is a target of miR-154-5p in cervical cancer. Further, the western blot assay showed that miR-154-5p overexpression inhibited the CUL2 protein expression, while miR-154-5p knockdown promoted the CUL2 protein expression. Meanwhile, our data showed a critical role for miR154-5p targeting CUL2 during cervical carcinogenesis, which is mediated through $\mathrm{pRb}$ expression. We showed that overexpression of miR-154-5p inhibited the oncogenic properties of SiHa cells via decreasing CUL2 and increasing pRb expression. Conversely, miR-154-5p knockdown promoted the oncogenic properties of SiHa cells via increasing CUL2 and decreasing $\mathrm{pRb}$ expression.

In conclusion, our study indicated that miR-154$5 p$ plays a crucial role in the inhibition of HPV16 E7-mediated $\mathrm{pRb}$ degradation by targeting the CUL2 3'UTR. Our results suggest that miR-154-5p is a potential target for the treatment of HPV-induced cervical cancer.

\section{Abbreviations}

CIN, cervical intraepithelial neoplasia; GO, Gene ontology; KEGG, Kyoto Encyclopedia of Genes and Genomes; TCT, ThinPrep cytologic test; NILM, negative for intraepithelial lesion or malignancy;
ASCUS, atypical squamous cells of undetermined significance; ASC-H, atypical cells of undetermined significance, cannot exclude a high-grade squamous intraepithelial lesion; LSIL, low-grade squamous intraepithelial lesion; HSIL, high-grade squamous intraepithelial lesion; SCC, squamous cell carcinoma; CUL2, Cullin 2; pRb, retinoblastoma protein; FIGO, International Federation of Gynecology and Obstetrics; ANOVA, analysis of variance.

\section{Supplementary Material}

Supplementary table.

http://www.jcancer.org/v11p5379s1.pdf

\section{Acknowledgements}

We thank Jing Li of Cnkingbio for bioinformatics assistance. We would like to thank Editage (www. editage.cn) for English language editing.

\section{Funding}

This research was supported by the National Natural Science Foundation of China [No.81702583, 81703313, 81972452]; the Outstanding Youth Fund Project of Shanxi Province [No.201901D211506]; the China Postdoctoral Science Foundation [No.2019M651072]; the Higher Education Discipline Innovation Project of Shanxi Province [No.20171104]; and the National Health and Family Planning Commission of the People's Republic of China [No. 201402010].

\section{Availability of data and materials}

The datasets used and/or analyzed during the present study are available from the corresponding author on reasonable request.

\section{Author Contributions}

WHZ conceived the study. WHZ, YTL collected and analyzed the data. YTL, YQL and HLZ performed the experiments. WHZ and LLZ provided the resources and supervised the study. WHZ and LD wrote the original draft. TW and $\mathrm{MH}$ reviewed and edited the manuscript. All authors read and approved the final manuscript.

\section{Competing Interests}

The authors have declared that no competing interest exists.

\section{References}

1. Siegel RL, Miller KD, Jemal A. Cancer statistics, 2019. CA Cancer J Clin. 2019; 69: 7-34.

2. McClung NM, Gargano JW, Bennett NM, et al. Trends in Human Papillomavirus Vaccine Types 16 and 18 in Cervical Precancers, 2008-2014. Cancer Epidemiol Biomarkers Prev. 2019; 28: 602-9.

3. Zhang C, Luo Y, Zhong R, et al. Role of polycyclic aromatic hydrocarbons as a co-factor in human papillomavirus-mediated carcinogenesis. BMC Cancer. 2019; 19: 138. 
4. Zhao W, Hao M, Wang Y, et al. Association between folate status and cervical intraepithelial neoplasia. Eur J Clin Nutr. 2016; 70: 837-42.

5. Huh K, Zhou X, Hayakawa H, et al. Human papillomavirus type 16 E7 oncoprotein associates with the cullin 2 ubiquitin ligase complex, which contributes to degradation of the retinoblastoma tumor suppressor. J Virol. 2007; 81: 9737-47.

6. White EA, Sowa ME, Tan MJ, et al. Systematic identification of interactions between host cell proteins and E7 oncoproteins from diverse human papillomaviruses. Proc Natl Acad Sci U S A. 2012; 109: E260-7.

7. Todorovic B, Hung $K$, Massimi $P$, et al. Conserved region 3 of human papillomavirus $16 \mathrm{E} 7$ contributes to deregulation of the retinoblastoma tumor suppressor. J Virol. 2012; 86: 13313-23.

8. Miroshnichenko S, Patutina O. Enhanced Inhibition of Tumorigenesis Using Combinations of miRNA-Targeted Therapeutics. Front Pharmacol. 2019; 10: 488

9. Arbyn M, Weiderpass E, Bruni L, et al. Estimates of incidence and mortality of cervical cancer in 2018: a worldwide analysis. Lancet Glob Health. 2020; 8: e191-e203.

10. Kjaer SK, Frederiksen K, Munk C, et al. Long-term absolute risk of cervical intraepithelial neoplasia grade 3 or worse following human papillomavirus infection: role of persistence. J Natl Cancer Inst. 2010; 102: 1478-88.

11. Cancer Genome Atlas Research N, Albert Einstein College of M, Analytical Biological S, et al. Integrated genomic and molecular characterization of cervical cancer. Nature. 2017; 543: 378-84.

12. Mirabello L, Yeager M, Yu K, et al. HPV16 E7 Genetic Conservation Is Critical to Carcinogenesis. Cell. 2017; 170: 1164-74 e6.

13. Songock WK, Kim SM, Bodily JM. The human papillomavirus E7 oncoprotein as a regulator of transcription. Virus Res. 2017; 231: 56-75.

14. Hoppe-Seyler K, Bossler F, Braun JA, et al. The HPV E6/E7 Oncogenes: Key Factors for Viral Carcinogenesis and Therapeutic Targets. Trends Microbiol. 2018; 26: 158-68.

15. Scott DC, Rhee DY, Duda DM, et al. Two Distinct Types of E3 Ligases Work in Unison to Regulate Substrate Ubiquitylation. Cell. 2016; 166: 1198-214 e24.

16. Wang S, Xia W, Qiu M, et al. Atlas on substrate recognition subunits of CRL2 E3 ligases. Oncotarget. 2016; 7: 46707-16.

17. Cai $\mathrm{W}$, Yang H. The structure and regulation of Cullin 2 based E3 ubiquitin ligases and their biological functions. Cell Div. 2016; 11: 7.

18. Su Y, Ni Z, Wang G, et al. Aberrant expression of microRNAs in gastric cancer and biological significance of miR-574-3p. Int Immunopharmacol. 2012; 13: $468-75$.

19. Xu J, Fang $\mathrm{Y}$, Wang $\mathrm{X}$, et al. CUL2 overexpression driven by CUL2/E2F1/miR-424 regulatory loop promotes HPV16 E7 induced cervical carcinogenesis. Oncotarget. 2016; 7: 31520-33

20. He Y, Lin J, Ding Y, et al. A systematic study on dysregulated microRNAs in cervical cancer development. Int J Cancer. 2016; 138: 1312-27.

21. Gomez-Gomez Y, Organista-Nava J, Gariglio P. Deregulation of the miRNAs expression in cervical cancer: human papillomavirus implications. Biomed Res Int. 2013; 2013: 407052.

22. Cardote TAF, Gadd MS, Ciulli A. Crystal Structure of the Cul2-Rbx1-EloBC-VHL Ubiquitin Ligase Complex. Structure. 2017; 25: 901-11 e3.

23. Ren $\mathrm{H}$, Ma X, Shao $\mathrm{Y}$, et al. Correlation Between Serum miR-154-5p and Osteocalcin in Males and Postmenopausal Females of Type 2 Diabetes With Different Urinary Albumin Creatinine Ratios. Front Endocrinol (Lausanne). 2019; 10: 542.

24. Bolandghamat Pour Z, Nourbakhsh M, Mousavizadeh K, et al. Suppression of nicotinamide phosphoribosyltransferase expression by miR-154 reduces the viability of breast cancer cells and increases their susceptibility to doxorubicin. BMC Cancer. 2019; 19: 1027.

25. Kai $Y$, Qiang C, Xinxin P, et al. Decreased miR-154 expression and its clinical significance in human colorectal cancer. World J Surg Oncol. 2015; 13: 195.

26. Wang L, Wu L, Wu J. Downregulation of miR-154 in human glioma and its clinicopathological and prognostic significance. J Int Med Res. 2016; 44: 994-1001.

27. Lin $X$, Yang $Z$, Zhang $P$, et al. miR-154 suppresses non-small cell lung cancer growth in vitro and in vivo. Oncol Rep. 2015; 33: 3053-60.

28. Pang $X$, Huang $K$, Zhang Q, et al. miR-154 targeting ZEB2 in hepatocellular carcinoma functions as a potential tumor suppressor. Oncol Rep. 2015; 34: $3272-9$.

29. Zhou H, Zhang Y, Lai Y, et al. Circ_101064 regulates the proliferation, invasion and migration of glioma cells through miR-154-5p/ PIWIL1 axis. Biochem Biophys Res Commun. 2020; 523: 608-14.

30. Chen J, Ma C, Zhang Y, et al. MiR-154-5p Suppresses Cell Invasion and Migration Through Inhibiting KIF14 in Nasopharyngeal Carcinoma. Onco Targets Ther. 2020; 13: 2235-46.

31. Tian $\mathrm{Q}, \mathrm{Gu} \mathrm{Y}$, Wang $\mathrm{F}$, et al. Upregulation of miRNA-154-5p prevents the tumorigenesis of osteosarcoma. Biomed Pharmacother. 2020; 124: 109884

32. Lin C, Li Z, Chen P, et al. Oncogene miR-154-5p regulates cellular function and acts as a molecular marker with poor prognosis in renal cell carcinoma. Life Sci. 2018; 209: 481-9.

33. Xu M, Chen $X$, Lin $K$, et al. The long noncoding RNA SNHG1 regulates colorectal cancer cell growth through interactions with EZH2 and miR-154-5p. Mol Cancer. 2018; 17: 141. 